\title{
Método de dissipação térmica para determinação do fluxo de seiva em coqueiro anão-verde
}

\author{
Marilaine Campanati Araújo; Barbara dos Santos Esteves (*); Elias Fernandes de Sousa \\ Universidade Estadual do Norte Fluminense (UENF), Laboratório de Engenharia Agrícola, Av. Alberto Lamego, 2000, 28013-602 \\ Campos dos Goytacazes (RJ), Brasil. \\ (*) Autora correspondente: barbbarase@yahoo.com.br
}

Recebido: 18/nov./2011; Aceito: 16/nov./2012

\begin{abstract}
Resumo
O conhecimento da demanda hídrica das culturas é uma informação básica para o manejo de irrigação. O método de dissipação térmica (MDT) vem recebendo atenção nos últimos tempos para esta finalidade, em virtude de utilizar princípios físicos relativamente simples e poder ser aplicado diretamente no campo. Este trabalho objetivou realizar a calibração do método de sonda de dissipação térmica na determinação do fluxo de seiva em coqueiro anão-verde. Para a calibração, a sonda foi inserida em um segmento de pecíolo da folha do coqueiro-anão verde. Um fluxo estável de água foi estabelecido no segmento do pecíolo. Foram feitas medidas de vazão, volume por unidade de tempo, e temperatura, dada pela sonda. Após a calibração em laboratório foram realizados experimentos de campo na Região Norte Fluminense, Brasil, nos quais o modelo calibrado foi capaz de acompanhar a demanda atmosférica. Conclui-se que modelo matemático obtido da calibração do MDT é satisfatório para a obtenção da transpiração no coqueiro anão-verde.
\end{abstract}

Palavras-chave: transpiração, manejo de água, método de Granier, Cocos nucifera L.

\section{The heat dissipation method for sap flow determination in green-dwarf coconut plants}

\begin{abstract}
The knowledge of crop water demand is basic for irrigation management. In recent years, the Heat Dissipation Method (HDM) has received attention for this purpose because its relatively simple physical principles and applicability in field conditions. This study aimed to perform calibration of the HDM for sap flow determination in green-dwarf coconut plants. For calibration, the probe was inserted in a segment of the leaf petiole of a dwarf coconut tree. A steady stream of water was established in the segment of petiole. Measurements of flow rate, volume per unit time and temperature, were taken with the probe. After calibrating HDM in laboratory, field experiments were carried out in the Norte Fluminense, Brazil, where the calibrated model were well coupled to the atmospheric demand. It is concluded that the mathematical model obtained in the HDM calibration is suitable for obtaining the transpiration to green dwarf coconut.
\end{abstract}

Key words: transpiration, water management, Granier method, Cocos nucifera L.

\section{INTRODUÇÃO}

O conhecimento das necessidades hídricas das culturas é informação básica para a tomada de decisão e o bom uso e a conservação dos recursos hídricos em culturas irrigadas (Oliveira et al., 2009), sendo a evapotranspiração uma informação essencial para este fim. Em cultivos do tipo pomar, nos quais a cobertura do solo é descontínua, a transpiraçáo é o principal elemento da evapotranspiração (VelLAME et al., 2010).

Grande parte dos estudos sobre transpiração de plantas, principalmente em fruteiras lenhosas, baseia-se em metodologias de fornecimento de calor no caule (Vellame et al., 2011), as quais pressupóem a equivalência entre o fluxo xilemático e o fluxo transpiratório foliar, em escala diária. FernÁndez et al. (2008) citam que as mediçóes do fluxo de seiva podem ser utilizadas para determinaçáo in situ do consumo hídrico das plantas, sendo sistemas facilmente automatizados, obtendo resultados satisfatórios para estimativa da transpiração.

Granier (1985) propôs o método de dissipação térmica (MDT) que consiste em sondas termoelétricas que são inseridas no tronco para a medição do fluxo de seiva. As medidas são realizadas de forma indireta, pois as sondas fornecem valores em milivoltagem, e o fluxo de seiva é expresso em densidade. As sondas para a medição do fluxo de seiva são compostas de duas agulhas de dois milímetros de diâmetro e três centímetros de comprimento, inseridas no caule, separadas por uma distância vertical de cinco centímetros. A agulha superior possui em seu interior uma resistência elétrica de constantã (elemento aquecedor) e um termopar 
de cobre-constantá (elemento medidor de temperatura). A agulha inferior contém apenas um termopar. Quando uma corrente elétrica é fornecida à resistência, esta se aquece e os termopares medem a diferença de temperatura entre as agulhas que é dependente do fluxo de seiva ocorrente durante a medição. Quando náo há fluxo de seiva, a diferença de temperatura é máxima; com o aumento do fluxo de seiva, o calor é dissipado mais rapidamente da fonte de aquecimento e a diferença de temperatura diminui. A densidade de fluxo de seiva é calculada através de uma relação empírica determinada por GrANIER (1985).

O MDT vem recebendo atenção nos últimos tempos em virtude de utilizar princípios físicos relativamente simples e poder ser aplicado diretamente no campo sem alterar as condições fisiológicas e micrometeorológicas da planta (Pimentel et al. 2010). Segundo Braun (1997), a grande vantagem do método de Granier é a facilidade de confecção e instalação do sensor, quando comparado com outros métodos térmicos. No entanto, apesar da simplicidade de confecção e instalação dos sensores do MDT, há a necessidade de estudá-lo mais profundamente, principalmente no que diz respeito à sua calibração para cada espécie de planta estudada, assim como a maneira de minimizar os potenciais erros mencionados na literatura (Delgado-Rojas et al., 2007). Os mesmos autores afirmaram que náo é garantido que a equação original de Granier (1985) possa servir para plantas tropicais, vendo-se, portanto, a necessidade de se realizarem localmente calibraçóes com plantas de interesse agrícola para o país. SMith e Allen (1996) salientam a importância do melhor entendimento das técnicas do fluxo de seiva a fim de selecionar o método mais apropriado para utilização, evitando erros.

Para o coqueiro anão-verde (Cocos nucifera L.) não há informaçôes sobre a calibração do método da dissipação térmica na localidade climática da Região Norte Fluminense, sendo escassas também informaçóes sobre sua demanda hídrica. Dessa forma, este trabalho objetivou a calibração do método de sonda de dissipação térmica na determinação do fluxo de seiva em coqueiro anão-verde.

\section{MATERIAL E MÉTODOS}

Granier (1985) propôs uma equação para " $u$ " (Equação 1), ajustada para diversas espécies arbóreas.

$u=119,003 K^{1,231}$

em que: $u$ é a densidade do fluxo de seiva $\left(10^{-6} \mathrm{~m}^{3} \mathrm{~s}^{-1} \mathrm{~m}^{-2}\right)$ e $K$ é o coeficiente de dissipação térmica, obtido pela Equaçáo 2, apresentada a seguir:

$K=\frac{\left(\Delta T_{\text {máx }}-\Delta T\right)}{\Delta T_{\text {máx }}}$ em que: $D T_{\text {máx }}$ é a Diferença de temperatura máxima, quando o fluxo de seiva é nulo; $D T$ é a Diferença de temperatura quando o fluxo de seiva é diferente de zero.

Para a calibração das sondas de dissipação térmica, foi construído em laboratório, um aparelho segundo o esquema apresentado por Fernández et al. (2001) e DelgadoRojas et al. (2006). Na calibração do método na cultura do coqueiro anão-verde foi utilizado um segmento de pecíolo e fixado no corpo do equipamento por um molde de silicone e parafusos. Uma sonda foi inserida no pecíolo e ligada ao coletor de dados.

O coletor de dados foi conectado a um microcomputador portátil que permitiu a observação simultânea dos valores fornecidos pela sonda, enquanto a vazão (Q) $\left(\mathrm{m}^{3} \mathrm{~s}^{-1}\right)$ no pecíolo foi medida com proveta graduada. As leituras foram feitas a cada minuto, registrando-se os dados em uma planilha. Os valores de vazão foram divididos pela área da seção do pecíolo, obtendo-se, então, a densidade de fluxo $\left(\mathrm{m}^{3} \mathrm{~m}^{-2} \mathrm{~s}^{-1}\right)$, que foi relacionada com os valores de DT fornecidos pela sonda. Realizaram-se cinco repetiçôes, sendo cada uma constituída de um segmento de pecíolo e uma sonda diferente. Em cada repetição, diversos valores de vazão e DT foram medidos. Com os dados das sondas na calibraçấo, calculou-se o coeficiente $K$.

Após a calibraçáo, o modelo proposto foi testado e validado no campo para obtençáo da transpiração da cultura. O experimento de campo foi realizado na Fazenda Taí, em Campos dos Goytacazes, RJ (214ㄱ'S; $41^{\circ} 17^{\prime} \mathrm{O}$; altitude de $11 \mathrm{~m}$ ), Região Norte Fluminense, Brasil, onde os coqueiros da variedade anão-verde foram plantados no espaçamento de 7,5 x 7,5 x 7,5 m, em distribuição triangular. $\mathrm{O}$ solo da área é arenoso, de origem aluvial. A evapotranspiração de referência foi calculada, segundo a equaçáo de Penman-Monteith parametrizada pela FAO (Allen et al., 1998). Os dados meteorológicos foram obtidos por uma estação automática da marca Thies Clima, modelo DL-15, instalada próximo ao local do experimento. A área foi irrigada pelo sistema de microaspersão, sendo colocados dois microaspersores por planta, com vazão de $30 \mathrm{~L} \mathrm{~h}^{-1}$.

No experimento de campo, selecionou-se uma planta sadia, de 3,5 anos de idade, com aproximadamente 22 folhas bem formadas e desenvolvidas. Nesta planta, foram escolhidas quatro folhas intermediárias totalmente expandidas, com inclinaçáo aproximada de $45^{\circ}$, cada uma localizada em um quadrante da planta, nas direções Norte, Leste, Sul e Oeste, para a inserção das sondas. Estas foram inseridas no pecíolo, a cerca de $30 \mathrm{~cm}$ da inserção do último folíolo. Todo o conjunto foi coberto por uma manta aluminizada para evitar a incidência de raios solares. As sondas foram ligadas ao coletor automático de dados, o qual realizava leituras a cada segundo e armazenava médias de $10 \mathrm{~min}$.

O experimento de medição de densidade de fluxo de seiva foi realizado entre 27 de outubro e 4 de novembro e 
$1 .^{\circ}$ e 13 de dezembro de 2002. No fim de cada período, a área foliar de cada folha utilizada foi medida por intermédio de medidor de área foliar LI-COR 1500. Relacionouse a média do volume diário transpirado relativo de cada folha ( $\mathrm{L} \mathrm{d}^{-1} \mathrm{~m}^{-2}$ de área foliar) com a evapotranspiração de referência $\left(\mathrm{mm} \mathrm{d}^{-1}\right)$.

Para avaliação da calibração, ajustou-se um modelo de regressão linear simples entre os valores de " $u$ " estimados pelo modelo obtido na calibraçáo e os valores preditos pela equação de Granier (1985). O ajuste entre os valores de transpiração e evapotranspiração de referência foi realizado a partir da análise de regressão linear segmentada, conforme Portz et al. (2000).

\section{RESULTADOS E DISCUSSÃO}

Os dados pareados de $K$ e " $u$ " (Figura 1), coletados para a calibração do MDT para a cultura do coqueiro-anão verde, apresentaram índices da análise de regressão linear significativos ao nível de $1 \%$ de probabilidade, com $\mathrm{R}^{2}$ de $0,956$. Cabibel e Do (1991), estudando quatro espécies de plantas e Köstner et al. (1998), com seis espécies de plantas, verificaram relaçóes muito próximas à de Granier. Assim, estes autores recomendam a utilização da equação original de Granier como padrão para qualquer planta lenhosa. Todavia, Smith e Allen (1996) salientam que os parâmetros utilizados na calibração da equaçáo de Granier não dependem das características das árvores ou da anatomia da madeira, mas se fundamentam nos princípios físicos que regem a transferência de calor. Assim, recomenda-se a calibração do método para espécies em que nunca foi validado, corroborando a necessidade de trabalhos de calibração para diferentes espécies, em diferentes períodos e localidades climáticas.

Comparando-se os valores diários de " $u$ ", estimados pelo modelo obtido na calibraçâo e os valores conforme



Figura 1. Relação entre os valores de coeficiente de dissipação térmica $(K)$ e os valores de densidade de fluxo de seiva $(u)$ obtidos na calibração do método de medição do fluxo de seiva para a cultura do coqueiro anão-verde. modelo apresentado por Granier (Figura 2), verificou-se a similaridade da ordem de grandeza dos coeficientes dos modelos matemáticos. Observou-se alta correlação entre os valores estimados pelos modelos. O coeficiente de inclinação foi estatisticamente igual à unidade e o coeficiente de interseção diferente de zero. $\mathrm{O}$ fato do coeficiente de interseção ser diferente de zero demonstra que existe uma diferença sistemática entre os modelos comparados. Provavelmente, a diferença pode ser decorrente do fato de que o modelo de Granier utilizou para o cálculo de " $u$ ", a área do xilema ativo, enquanto no modelo de calibraçáo utilizou-se toda a seçáo do pecíolo, onde o xilema está uniformemente distribuído.

A definição da área condutora da seiva, ou seja, a área transversal ocupada pelo xilema, no ponto onde está inserido o sensor, que é efetiva em transportar a água, constitui-se em uma grande fonte de erro do MDT. Esta área depende principalmente da espécie vegetal, da idade da árvore e do diâmetro do caule (Delgado Rojas et al., 2006). Pode-se verificar na literatura que, comumente, para estimativa da densidade de fluxo de seiva, segundo a proposta da Granier (1985), as sondas são inseridas no tronco da planta em estudo (Pimentel et al., 2010; Delgado Rojas et al., 2006; Naves-Barbiero et al., 2000; Lu e Снаско, 1998). Testes preliminares não forneceram resultados satisfatórios de medição da densidade de fluxo de seiva, quando as sondas foram inseridas no tronco do coqueiro anão-verde, provavelmente devido ao pequeno comprimento das agulhas em relação ao diâmetro do tronco e à diferente distribuição do tecido xilemático entre liliopsidas, como o coqueiro anão-verde, e magnoliopsidas, as quais foram estudadas pelos autores citados acima. Clearwater et al. (1998) citam que erros podem ocorrer se os sensores estiverem em contato com áreas do xilema que não conduzem a seiva, como o cerne e a casca, podendo gerar subestimativas da velocidade do fluxo.

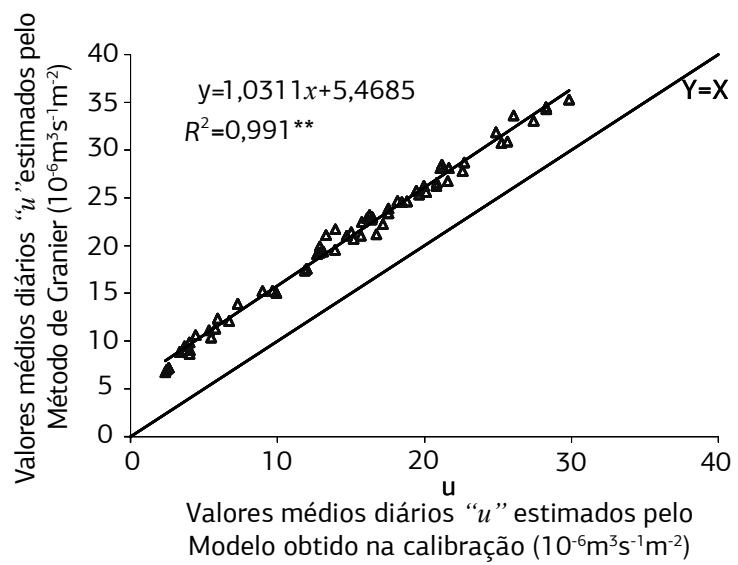

Figura 2. Relação entre valores médios diários de densidade de fluxo de seiva $(u)$ obtidos pelo modelo de Granier (1985) e os obtidos pelo modelo calibrado. 
O modelo calibrado para a cultura do coqueiro anão-verde acompanhou as variaçôes climáticas do período de estudo, demonstrando que a relação entre transpiração e evapotranspiração de referência do coqueiro anão-verde não foi constante no período avaliado, ou seja, a relação não apresenta uma taxa de incremento única, sendo variável em função da demanda atmosférica (Figura 3). Observou-se em campo que a transpiração média das plantas avaliadas durante o período de estudo, utilizando-se o modelo calibrado (Figura 1), foi de $0,96 \mathrm{~L} \mathrm{~d}^{-1} \mathrm{~m}^{-2}$ de área foliar, com o mínimo de 0,09 e o máximo de $1,52 \mathrm{~L} \mathrm{~d}^{-1} \mathrm{~m}^{-2}$ área foliar. Pimentel et al. (2010), com a equação calibrada para o cafeeiro, obtiveram valores de transpiração para as condiçôes de seu estudo, bem como fizeram comparaçóes com os elementos meteorológicos para identificar aqueles que mais afetam no consumo hídrico das plantas. Os mesmos autores observaram que houve concordância entre os valores de transpiraçáo e evapotranspiração de referência, para a maioria das plantas analisadas em sua pesquisa.

Para o coqueiro anáo-verde, nos valores de evapotranspiração de referência de $1,8 \mathrm{~mm} \mathrm{~d}^{-1}$ até aproximadamente $4,1 \mathrm{~mm} \mathrm{~d}^{-1}$, observou-se que a transpiração e a evapotranspiraçáo de referência relacionaram-se linearmente, demonstrando aumento da primeira diretamente em resposta à demanda atmosférica. Em outra análise, quando a evapotranspiração de referência atingiu valores maiores que $4,1 \mathrm{~mm} \mathrm{~d}^{-1}$, a transpiração tendeu a se estabilizar. Vellame et al. (2012), estudando a laranjeira Valência, observaram que a relação entre a transpiração e a evapotranspiração de referência não foi linear em toda a faixa de demanda evaporativa da atmosfera, variando em função da disponibilidade energética do período de estudo. Girardi et al. (2010) observaram em árvores de citros que houve diminuição temporária do fluxo de seiva nos períodos de máximo estresse hídrico, usualmente observado ao meio-dia, sendo este fato possivelmente atribuído ao fechamento estomático.

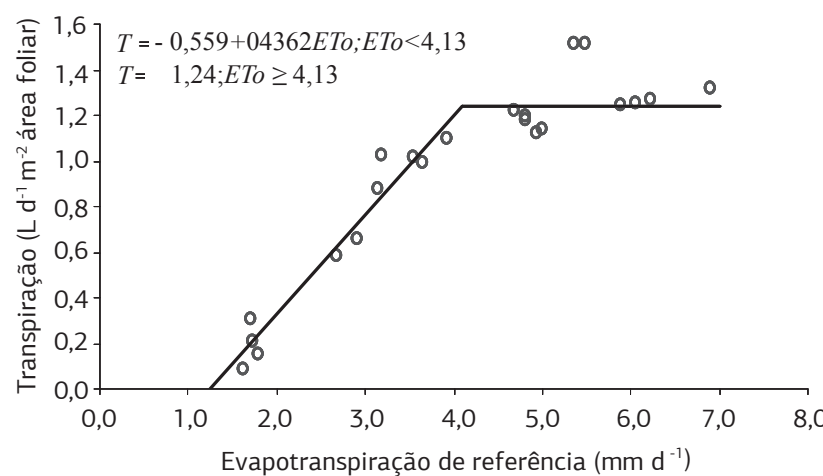

Figura 3. Relação entre Evapotranspiração de Referência (ETo), em $\mathrm{mm} \mathrm{d}^{-1}$, e a Transpiração $(\mathrm{T})$ do coqueiro-anão de 3,5 anos de idade, em L d ${ }^{-1} \mathrm{~m}^{-2}$ área foliar, no Norte Fluminense.

\section{CONCLUSÃO}

O modelo matemático obtido da calibração do método de dissipação térmica para o coqueiro anão-verde é satisfatório para a obtenção dos valores do fluxo de seiva, e por conseguinte, para estimativa da transpiraçáo da cultura.

\section{REFERÊNCIAS}

ALLEN, R.G.; PEREIRA, L.S.; RAES, D.; SMITH, M. Crop evapotranspiration: Guidelines for computing crop water requirements. FAO: Rome, 1998. 310p. (FAO Irrigation and Drainage Paper 56)

BRAUN, P. Sap flow measurements in fruit trees - Advantages and shortfalls of currently used systems. Acta Horticulturae, n. 449, p.267-272, 1997.

CABIBEL, B.; DO, F. Mesures thermiques des flux de sève dans les troncs et les racines et fonctionnement hydrique des arbres: I. Analyse théorique des ereurs sur la mesure des flux et validation des mesures en présence de gradients thermiques extérieurs. Agronomie, v.11, p.669-678, 1991.

CLEARWATER, M.J.; MEINZER, F.C.; ANDRADE, J.L.; GOLDSTEIN, G.; HOLBROOK, N.M. Potential errors in measurement of nonuniform sap flow using heat dissipation probes. Tree Physiology, v.19, p.681-687, 1998.

DELGADO-ROJAS, J.S.; ANGELOCCI, L.R.; FOLEGATTI, M.V.; COELHO FILHO, M.A. Desempenho da sonda de dissipaçáo térmica na medida da transpiraçáo de plantas jovens de lima ácida. Engenharia Agrícola, v.27, p.404-413, 2007.

DELGADO-ROJAS, J.S.; RIGHI, C.A.; KARASAWA, S.; ANGELOCCI, L.R.; BERNARDES, M.S.; FOLEGATTI, M.V. Desempenho do método de dissipação térmica na medida do fluxo de seiva em seringueira. Engenharia Agrícola, v.26, p.722-729, 2006.

FERNÁNDEZ, J.E.; GREEN, S.; CASPARI, H.; DIAZ-ESPEJO, A.; CUEVAS, M. The use of sap flow measurements for scheduling irrigation in olive, apple and Asian pear trees and in grapevines. Plant and Soil, v.305, p.91-104, 2008.

FERNÁNDEZ, J.E.; PALOMO, M.J.; DI'AZ-ESPEGJO, A.; CLOTHIER, B.E.; GREEN, S.R.; GIRO’ N, I.F.; MORENO, F. Heat-pulse measurements of sap flow in olives for automating irrigation: tests, root flow and diagnostics of water stress. Agricultural Water Management, v.51, p.99-123, 2001.

GIRARDI, E.A.; MOURÃO FILHO, F.A.A.; DELGADOROJAS, J.S.; ARAÚJO, J.P.C. Use of the heat dissipation method for sap flow measurement in citrus nursery trees. Revista Brasileira Fruticultura, v.32, p.976-983, 2010.

GRANIER, A. Une nouvelle méthode pour la mesure du flux de sève brute dans le tronc des arbres. Annales des Sciences Forestières, v.42, p.193-200, 1985.

LU, P.; CHACKO, E. Evaluation of Granier's sap flux sensor in Young mango trees. Agronomie, v.18, p.461-471, 1998. 
NAVES-BARBIERO, C.C.; FRANCO, A.C.; BUCCI, S.J.; GOLDSTEIN, G. Fluxo de seiva e condutância estomática de duas espécies lenhosas sempre-verdes no campo sujo e cerradão. Revista Brasileira de Fisiologia Vegetal, v.12, p.119-134, 2000.

OLIVEIRA, G.X.S.; COELHO FILHO, M.A.; PEREIRA, F.A.C.; PAZ, V.P.S.; CASTRO NETO, M.T. Relaçóes entre transpiração máxima, evapotranspiração de referência e área foliar em quatro variedades de mangueira. Revista Brasileira de Fruticultura, v.31, p.20-27, 2009.

PIMENTEL, J.S.; SILVA, T.J.A.; BORGES JÚNIOR, J.C.F., FOLEGATTI, M.V., MONTENEGRO, A.A.A. Estimativa da transpiração em cafeeiros utilizando-se sensores de dissipação térmica. Revista Brasileira de Engenharia Agrícola e Ambiental, v.14, p.187-195, 2010.

PORTZ, L.; DIAS, C.T.S.; CYRINO, J.E.P. Regressão segmentada como modelo na determinaçáo de exigências nutricionais de peixes. Scientia Agricola, v.57, p.601-607, 2000.
SMITH, D.M.; ALLEN, S.J. Measurement of sap flow in plants stems. Journal of Experimental Botany, v.47, p.1833$1844,1996$.

VELlAME, L.M.; COELHO, R.D.; TOLENTINO, J.B. Transpiração de plantas jovens de laranjeira 'Valência' sob porta-enxerto limão 'Cravo' e citrumelo 'Swingle' em dois tipos de solo. Revista Brasileira de Fruticultura, v.34, p.24-32, 2012.

VELLAME, L.M.; COELHO FILHO, M.A.; PAZ, V.P.S.; COELHO, E.F. Gradientes térmicos naturais na estimativa do fluxo de seiva pelo método Granier. Revista Caatinga, v.24, p.116-122, 2011.

VELLAME, L.M.; COELHO FILHO, M.A.; PAZ, V.P.S.; COELHO, E.F. Stem heat balance method to estimate transpiration of young orange and mango plants. Revista Brasileira de Engenharia Agrícola e Ambiental, v.14, p.594599, 2010. 